\title{
A Hospital Based Study on Female Genital Tuberculosis in Central India
}

\author{
Patel $\mathbf{S}^{1}$, Dhand PL ${ }^{2}$ \\ ${ }^{1}$ Dr. Swati Patel, Assistant Professor, ${ }^{2}$ Dr. P. L. Dhand, Professor \& Head , Department of Pathology, R. D. Gardi \\ Medical College, Ujjain, M.P, India.
}

Address for Correspondence: Dr. Swati Patel, B 6/4, RDGMC Campus, Ujjain, M.P, Email drswatipatelsoni02@gmail.com

\begin{abstract}
Introduction: In developing countries like India tuberculosis (TB) is common and still a major cause of infertility. Female genital tuberculosis is one form of extra pulmonary TB. Genital TB may be asymptomatic and diagnosis requires a high index of suspicion. Early diagnosis may improve the outcome before permanent tissue damage get established. The objective of this study was to evaluate the prevelance and clinico-pathological aspects and diagnosis of female genital tuberculosis (FGTB). Method: A total of 21 cases of female genital tuberculosis were diagnosed from 1672 patients over a period of 2 years, studied from January 2013 to December 2015. The diagnostic procedure used were endometrial curettage and biopsy, histopathological examination, culture, stain for Acid fast bacilli in correlation with clinical presentation, organ involvement, hysterosalpingography(HSG), Ultrasonography (USG)and laparoscopy. Most of the specimens received were endometrial curettage and biopsies. Result: Female genital TB accounted for $1.25 \%$ of all tuberculosis patients in this study. The highest incidence was between 21-30 years of ages, but the patient ranged from 18-60 years. FGTB involves the endometrium (16), fallopian tube (4),cervix (1). Out of all cases, 7 demonstrate typical epitheloid granuloma and rest with atypical tuberculous lesion, acid-fast bacilli in tissue section were detected in 3 cases of endometrium, 1 case of fallopian tube and culture positive in 3 cases of endometrium, 1 case of fallopian tube. Conclusion: Female genital tuberculosis is not uncommon in developing countries like India and indicates a strong relation between genital TB and infertility. The diagnosis of FGTB is challenging. Therefore, if possible genital TB is to be frequently diagnosed and considered in differential diagnosis of causes of infertility, in correlation with clinical and other diagnostic modalities.
\end{abstract}

Keywords: Tuberculosis (TB), Female genital tuberculosis (FGTB), Female infertility, Histopathology.

\section{Introduction}

In developing countries like India tuberculosis (TB) is common. Female genital tuberculosis is one form of extra pulmonary TB. It is by no means uncommon, particularly in communities where pulmonary or other forms of extragenital TB are common. Genital TB may be asymptomatic and diagnosis requires a high index of suspicion. Prevelance of genital tuberculosis world wide is between $5 \%$ and $10 \%$, while in India it is 19\% [1]. Genital tuberculosis is responsible for $5 \%$ of all pelvic infections and occurs in $10 \%$ cases of pulmonary tuberculosis [2]. The disease mainly affects individual below 40 years of age, with peak age range between 21-

Manuscript received: $01^{\text {st }}$ Feb 2016

Reviewed: $10^{\text {th }}$ Feb 2016

Author Corrected: $16^{\text {th }}$ Feb 2016

Accepted for Publication: $25^{\text {th }}$ Feb 2016
30 years of age [3]. Female genital tuberculosis is most often silent and sometime causes chronic pelvic inflammatory disease, menstrual disturbances, infertility or diagnosed after laparotomy for tubo-ovarian mass. It is one of the major etiological factors of female infertility. It has been estimated that approximately 5 $10 \%$ of females presenting to subfertility clinics worldwide have genital TB [2]. Prevelance of genital tuberculosis is much higher than one might imagine, as based on lack of report available in the literature, it may account for significant amount of female infertility [4].

Genital tuberculosis frequently involves fallopian tube in almost all cases followed by endometrium and cervix. It is mostly secondary infection acquired by 
hematogenous spread from an extragenital source in the lung, lymph node, urinary tract, bones, joints and bowel but direct spread from other intraperitoneal foci is very rare [5].

Diagnosis of early TB is very difficult. Diagnosis is often limited to clinical suspicion in countries where facilities for mycobacterium culture and histopathology are not available [6]. Abdominal and vaginal examinations may be normal. A high erythrocyte sedimentation rate and a positive mantoux test are nonspecific [7].

The diagnosis of tuberculosis from biopsy tissue depends on the demonstration of typical epitheloid granuloma and tissue reaction in those having tuberculosis may at times be atypical [8]. Many non tuberculous lesions can also produce granulomas mimicking tuberculosis. It is universally agreed that AFB are occasionally found in the endometrial and cervical granulomas and culture of menstrual blood for AFB is rarely positive [9]. Therefore, the true incidence of genital TB at an early stage cannot be assessed, owing to its subtle presentation; many cases remain undiagnosed [1].

\section{Objectives}

The study was conducted to evaluate the prevelance, clinico-pathological aspects and diagnosis of female genital tuberculosis (FGTB).

\section{Methods}

A retrospective and prospective study was done over a period of 2 years in the pathology department of our institute from January 2014 to December 2015. Present study included female patients of age group between 18-60 years, suspected of having genital tuberculosis, female factor of infertility, or having past and present contact history of tuberculosis and having abdominal pain. Detailed clinical information, radiologic and other relevant investigation were recorded from case sheets. The clinical information included age of the patient, demographic particulars, signs and symptoms with particular reference to the onset and duration of gynaecological illness, symptomatology like pelvic pain and irregular menstrual bleeding, scanty menstruation and amenorrhea. A pelvic mass in variable combination aroused a suspicion. History of contact with a tuberculosis cases, immunization status. Radiologic investigations included X-ray, ultrasonography (USG) of abdomen/pelvis, hysterosalpingography (HSG) and computed tomography scan and information regarding other relevant investigations like Mantoux test, erythrocyte sedimentation rate etc. were also recorded

Diagnostic procedure used to detect lesions were mostly histopathological examination of endometrial curettage and biopsy (13 cases), culture and Zeihl Neelsen stain. Patient presented with tubal involvement (4 cases), or underwent laparotomy for tubo-ovarian mass (1 case) and in 3 cases total hysterectomy specimen were submitted. HSG was done in 16 cases and USG in 9 cases. Exclusion criteria were females with diagnosis of other causes of infertility. Patients with granulomas on biopsy but later on proved to be non-tuberculosis by detection of Mycobacterium tuberculosis either in tissue section (ZN stain), culture or other diagnostic investigations.

Adequate biopsy tissue if possible had been sent for culture in Lowenstein Jensen medium. The remaining specimen had been examined after making paraffin embedded sections, stained by standard Haematoxylin and Eosin as well as Zeihl Neelsen stain. Acid fast bacilli were searched under the oil immersion lens. Periodic Acid Schiff and other special stains were done whenever necessary to exclude fungal etiology.

Diagnosis of FGTB was established by typical epitheloid granulomas in histopathologic examination (which is must) or positivity by AFB in tissue section, culture, PPD, HSG, USG, Laparoscopy etc. and clinical features. We preferred to combine all diagnostic tests together along with clinical correlation and epitheloid granulomas for making a diagnosis of FGTB, rather depending on a single diagnostic test [10,11].

\section{Result}

In total of 1672 gynaecological specimen selected for this study, 21(1.25\%) were diagnosed as having genital tuberculosis, The distribution of age shown in Table 1 ranged from $10-60$ years with the highest number in the age group 21-30 years. 
Table-1: Distribution of site of tuberculous specimens according to age of patients

\begin{tabular}{|c|c|c|c|c|c|}
\hline Age (years) & Endometrium & F.tubes/Ovaries & Cervix & Vulva & Total \\
\hline $10-20$ & 2 & 1 & - & - & 3 \\
\hline $21-30$ & 7 & 2 & 1 & - & 10 \\
\hline $31-40$ & 4 & 1 & - & - & 5 \\
\hline $41-50$ & 1 & - & - & - & 1 \\
\hline $51-60$ & 2 & - & - & - & 2 \\
\hline Total & 16 & 4 & 1 & & 21 \\
\hline
\end{tabular}

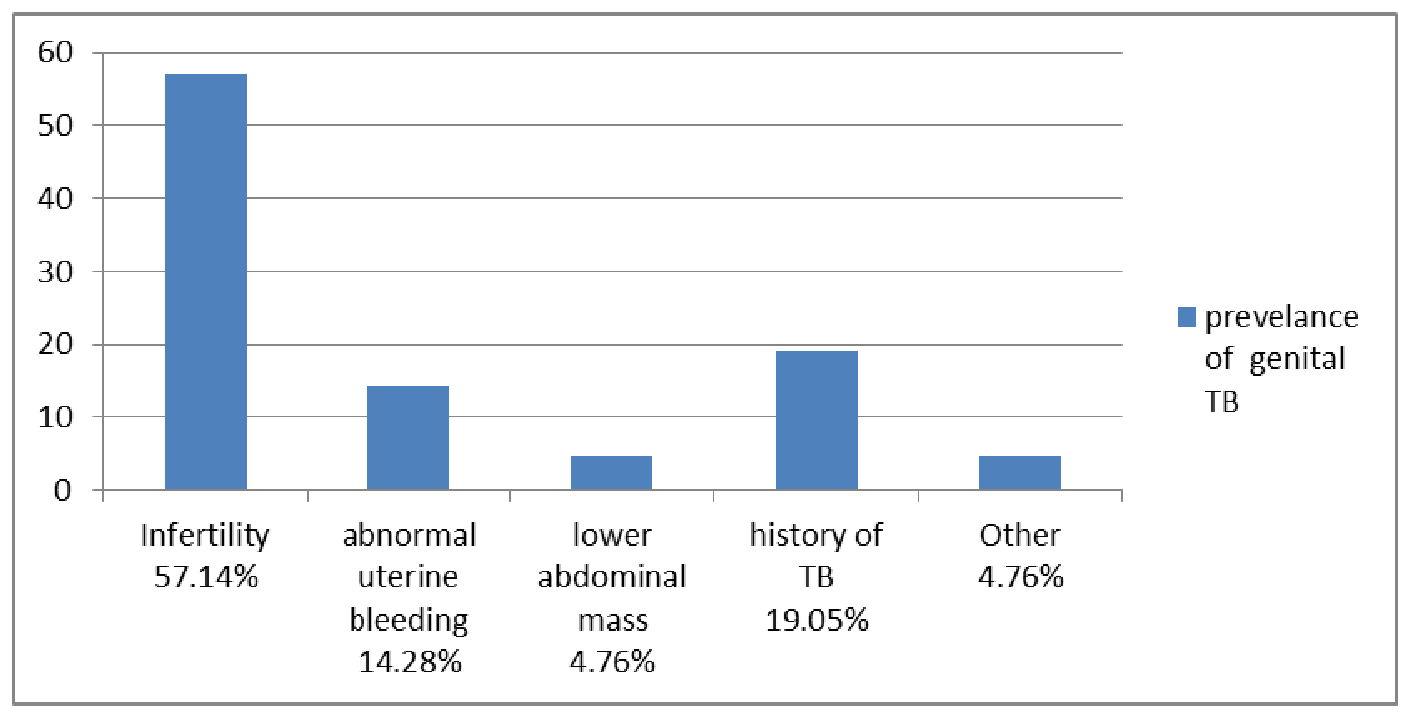

Figure 2: Mode of presentation of women with genital TB

Figure 2 show that $12(57.14 \%)$ patients had complained of infertility which was the commonest cause, menstural disturbances in 3 patient (14.28\%), 4 patients have history of tuberculosis (19.05\%), one case presented with pelvic/abdominal pain (4.76\%), one patient underwent laparotomy for tubo-ovarian mass (4.76\%) and one patient had record of abortion. Hysterosalpingography (HSG) was performed in 16 patients which revealed abnormality in either fallopian tube (4 cases) and endometrial cavity ( 2 cases). The findings in fallopian tube were tubal dialation (2/4), irregularities of tubal outline (1/4) and peritubal adhesions (1/4). Endometrial cavity occlusion was detected in two cases. Ultrasonography was done in 9 cases and findings were dialated/irregular fallopian tube (4/9), adnexal mass (1/9).

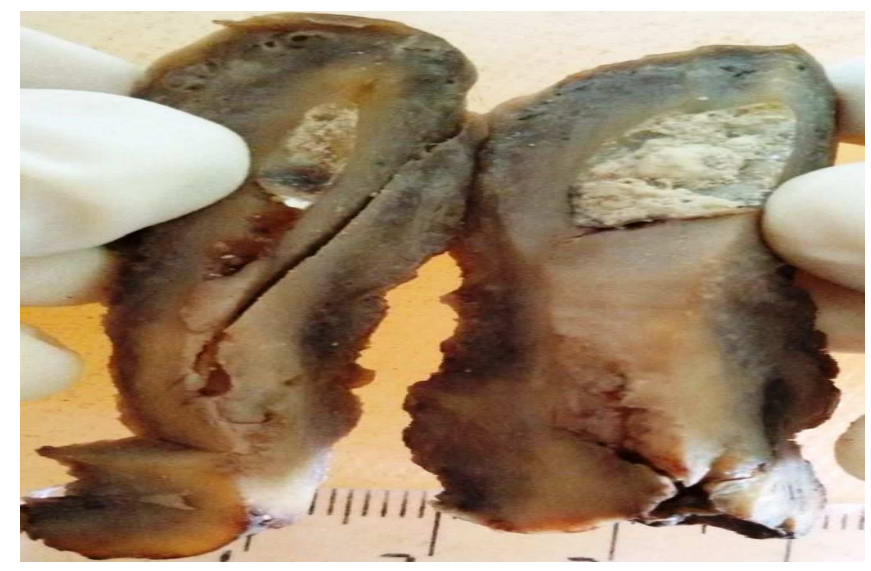

Figure 3: Gross photograph showing caseous material in endometrial cavity 
Gross appearance of the different specimen received for examination were usually unhealthy looking endometrium/cervix (3 cases)(figure 3) showing necrosis(cheesy), hemorrhage. The specimens of fallopian tubes (4 cases) were irregular due to adhesions, enlarged, edematous with thickened walls and areas of hemorrhages. No cases of TB in vulva and vagina were seen in this study.

Of the 21 tuberculous specimens, the most common diagnostic procedure was endometrial curettage and the main histologic finding in endometrial tuberculosis was the presence of small to medium sized epitheloid cell granulomas in different stages in functionalis layer. Figure $4 \& 5$ shows Langhans and foreign body type gaint cells in (6/16) cases of endometrium, while rest showed atypical tuberculous lesions with disruption of endometrial glands, non-specific inflammatory granulation with abundant plasma cells. Caseation was found very rarely in endometrium (one case as shown in figure 3). AFB were also rarely detected (3/16), culture positive in 3 cases and history of TB with PPD positive in 3 cases.
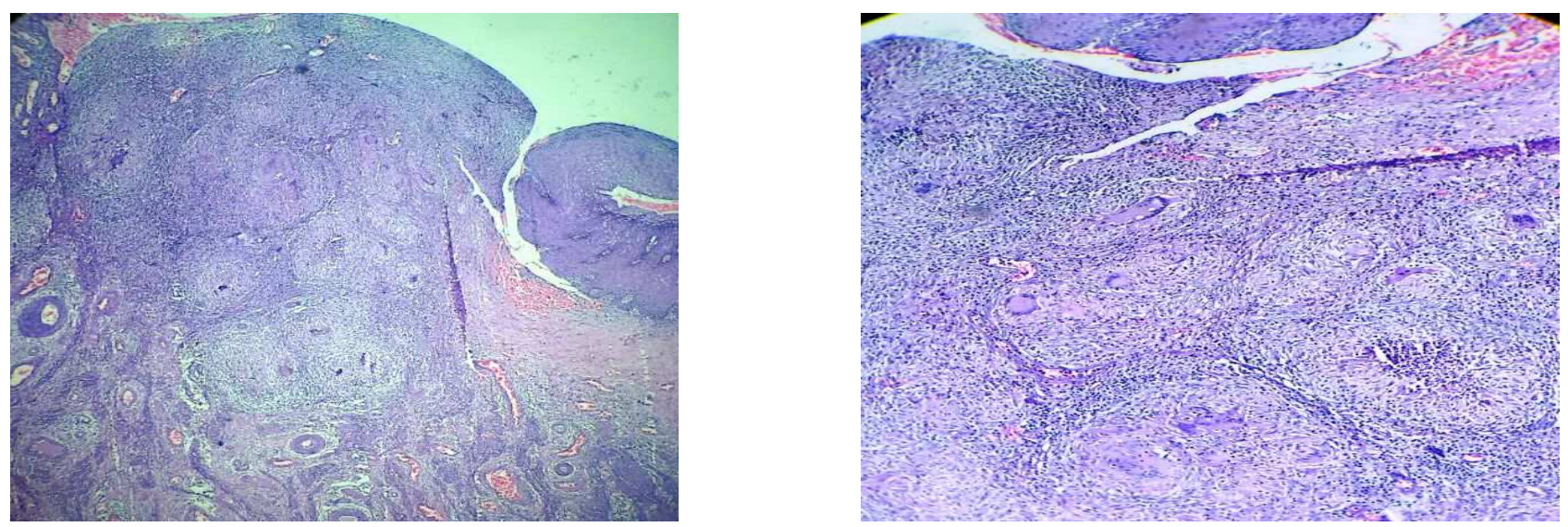

Figure 4 \& 5: Photomicrograph showing multiple epitheloid cell granulomas and Langhans gaint cell in functionalis layer of endometrium [H and E x100, x400]

Fallopian tube showed features of chronic salpingitis with non caseating granulomas (3/4) and typical granuloma in one case. AFB in tissue section were detected in (1/4) cases, culture positive in one case , and with history of TB in one case.Cervical tuberculosis shows epitheloid granulomas involving mucosa of endocervical canal but without caseation and AFB absent.

\section{Discussion}

Tuberculosis is a major global health problem. Actual frequency of female genital tuberculosis is unknown, despite various studies, as it is asymptomatic and often discovered incidentally [1]. The prevelance of female genital tuberculosis in our study found to be $1.25 \%$, similar to previous study in India by Srinivas reported in $2.08 \%$ [12]. Study done by Muechler et al shows FGTB patients are mostly in the reproductive age group, with highest number in age group 21-30 years, this is similar to our study [13].

The most common presentation reported earlier in the study by Carter et al, were infertility (44\%), pelvic pain (25\%), vaginal bleeding (18\%), amenorrhoea (5\%), vaginal discharge $(4 \%)$ and post menopausal bleeding
(2\%). Less common were ascites, tubo-ovarian mass, abdominal mass, abdominal distension [14]. In our study, similar results were obtained that (57.14\%) patients didnot show any symptoms and was diagnosed during study performed to evaluate the cause of their infertility. Clinically other different kinds of symptoms seen in the female are abnormal uterine bleeding (14.28 $\%$ ), lower abdominal mass i.e TO mass $(4.76 \%)$, other $(4.76 \%)$.

In this study Past, present and contact history of tuberculosis was seen in $(19.05 \%)$ of cases. Study by Shukla et al reported similar result that $20 \%$ of the patients with genital TB had history of TB in their family [15]. 
In most of the studies, most commonly involved site is the mucosa of fallopian tubes (92-100\%) with or without involvement of uterus and ovaries, endometrium $(50 \%)$, ovaries $(10-30 \%)$, cervix $(5 \%)$, vagina and vulva $(<1 \%)$ [16]. However, in our study endometrium was the most common site $16(76.19 \%)$ followed by fallopian tube $4(19.04 \%)$ and the remaining one $(4.76 \%)$ from cervix. Study by Mondal et al reported similar result [12]. The higher incidence of endometrium might be explained in our study, as most of the specimen we received was endometrial curettage for diagnostic work-up in infertile women.

In the present study, based on histopathologic criteria of tissue specimen, we found $33.3 \%$ of cases were positive and $66.66 \%$ were negative and this negative result was higher than reported by Abdulhakim as $31.1 \%$ [17]. The reason behind this negative result could be explained by obtaining the sample on the correct day of menstrual cycles. Inspite of negative result, we found $19.05 \%$ cases shows positive tubal involvement with HSG and $14.28 \%$ cases shows AFB in tissue section which was high, as compared to study by Mondal [12]. This might be explained by sample bias as it is small.

In tuberculous endometritis, caseation is rare in women of reproductive age group [18]. In our study also, caseation was found in only one woman who is postmenopausal similar to the study by Mondal [12]. The reason behind this is that tuberculous granuloma has to regenerate from the basal layer after menstrual shedding of the functionalis layer and the granulomas become well developed as the cycle progress. So, the biopsy is recommended in the late secretory or before mensturation. In postmenopausal women granulomas get enough time to develop caseation, as there is no periodic loss of endometrium.

In this study, AFB culture on Lowenstein Jensen (LJ) medium was positive in $14.28 \%$ cases. This finding is similar to the previous study that reported a positive culture in $15.6 \%$ of cases by Jindal [19] but lower than reported by Kashyap as $25 \%$ [20]. Although bacteriological isolation of mycobacteria is being considered the gold standard test [21], we found AFB culture as most unreliable test, reason behind is that, it was slow and requires 8 weeeks to grow with low sensitivity rate of $30-35 \%$. So because of this limitation in practical utility, we think a more rapidly technique and corerrelation with other diagnostic modalities is required.
This finding indicates that many tests are often required to obtain collective evidence for the diagnosis of GTB. Genital TB requires a high index of suspicion on the basis of clinical presentation, radiological findings(HSG,USG etc) and other investigations like Mantoux test, erythrocyte sedimentation rate and tuberculosis foci on chest X-ray in correlation with histopathological analysis, AFB in tissue section and culture.

\section{Conclusions}

Female genital tuberculosis is not uncommon in developing countries like India and indicates a strong relation between genital TB and infertility. The diagnosis of FGTB is challenging. Therefore, if possible genital TB is to be frequently diagnosed and considered in differential diagnosis of causes of infertility, in correlation with clinical and other diagnostic modalities. Though fallopian tube was the commonest site in many studies, in this study endometrium was more common.

In most of the other studies [22-24] too, and in our study the frequency of FGTB was low as typical epitheloid granuloma and demonstration of AFB by culture is low. Thus, histological diagnosis from tissue examination is only suggestive and not confirmatory. Therefore, different diagnostic technic should be combined judiciously and correlated with the clinical profile prior to instituting the antituberculosis treatment (ATT).

\section{Funding: Nil}

Conflict of interest: None.

Permission of IRB: Yes

\section{References}

1. Schaefer G. Tuberculosis of the female genital tract. Clin Obstet Gynecol. 1970 Dec;13(4):965-98.

2. Roya R, Sreenivasagari R, Cheruvu N R :Evaluation of women with infertility and genital tuberculosis. J Obstet Gynecol India 2006; 56: 423-426.

3. Nwachokor FN, Thomas JO. Tuberculosis in Ibadan, Nigeria--a 30 year review. Cent Afr J Med. 2000 Nov;46(11):287-92.

4. Namavar Jahromi B, Parsanezhad ME, GhaneShirazi R. Female genital tuberculosis and infertility. Int J Gynaecol Obstet. 2001 Dec;75(3):269-72. 
5. Gupta N, Sharma JB, Mittal S, Singh N, Misra R, Kukreja M. Genital tuberculosis in Indian infertility patients. Int J Gynaecol Obstet. 2007 May;97(2):135-8. Epub 2007 Mar 23.

6. Winfred J. Female genital tuberculosis. J Obstet Gynecol 1977; 63:418-28.

7. Arora VK, Gupta R, Arora R. Female genital tuberculosis: need for more research .Indian $\mathrm{J}$ Tuberc 2003;50(9): 9-11.

8. Chakraborty S, Chakraborty AK, Patra SP, Bhattacharya SK. Demonstration of acid-fast bacilli in tissues and evaluation of atypical tuberculous lesions. J Indian Med Assoc. 1993 Feb;91(2):30-3.

9. Agarwal J, Gupta JK. Female genital tuberculosis--a retrospective clinico-pathologic study of 501 cases. Indian J Pathol Microbiol. 1993 Oct;36(4):389-97.

10. Prasad S, Singhal M, Negi SS, Gupta S, Singh S, Rawat DS, Rai A. Targeted detection of $65 \mathrm{kDa}$ heat shock protein gene in endometrial biopsies for reliable diagnosis of genital tuberculosis. Eur $\mathrm{J}$ Obs Gynecol Reprod Biol 2012; 160: 215-218.

11. Mondal SK. Histopathologic analysis of female genital tuberculosis: a fifteen-year retrospective study of 110 cases in eastern India. Turk Patoloji Derg. 2013;29(1):41-5.

12. Srinivas Rao MS, Pavani K, Uma M, Krishna K, Vinayaraj EV, Dass M.. To evaluate the prevalence of female genital tuberculosis in Hyderabad . Int J Res Med Sci. 2013 Nov;1(4):421-423.

13. Muechler E, Minkowitz S. Postmenopausal endometrial tuberculosis. Obstet Gynecol. 1971 Nov;38(5):768-70.
14. Carter JR. Unusual presentations of genital tract tuberculosis. Int J Gynaecol Obstet. 1990 Oct;33(2):171-6.

15. Shukla S, Acharya N, Acharya S, Rajput DP, Vagha S. Fictitious pseudo Meig's syndrome: A medical emergency. J College Med Sci-Nepal 2011; 7: 57-64.

16. Varma TR. Genital tuberculosis and subsequent fertility. Int J Gynaecol Obstet. 1991 May;35(1):1-11.

17. Abdulhakim A Al, Ahmed S A, Abdelrahman H A $\mathrm{H}$ Genital tuberculosis is common among females with tubal factor infertility: Observational study'. Alexandri Journal of Medicine 2015; 51:321-324.

18. Roy A, Mukherjee S, Bhattacharya S, Adhya S, Chakraborty P. Tuberculous endometritis in hills of Darjeeling: a clinicopathological and bacteriological study. Indian J Pathol Microbiol. 1993 Oct;36(4):361-9.

19. Jindal UN. An algorithmic approach to female genital tuberculosis causing infertility. Int $\mathrm{J}$ Tuberc Lung Dis. 2006 Sep;10(9):1045-50.

20. Kashyap B, Kaur T, Jhamb R, Kaur IR Evaluating the utility of menstural blood versus endometrial biopsy as a clinical sample in the diagnosis of female genital tuberculosis. Am J Med Res 2012; 1(2):45-49.

21. Matthys H B, Frederick H, Vander M. Female genital tuberculosis : CPD S Afr Fam Pract 2008; 50(5):12-16.

22. Mukta K, Kher A., Sharma K.D. Tuberculosis of endometrium - A histopathological study of 385 cases; Ind. J. Pathol Microbiol, 1997; 20: 39.

23. Devi P.D. Genital tuberculosis in the female; JTMA, 1962; 38:164.

24. Sammel K.C, Gupta P. Tuberculous endometritis. J Obstet Gynae India, 1967; 17: 14.

\section{How to cite this article?}

Patel S, Dhand PL A Hospital Based Study on Female Genital Tuberculosis in Central India. Int J Med Res Rev 2016;4(2):227-232. doi: 10.17511/ijmrr.2016.i02.017. 